\title{
A Framework for Distributed Resource Allocation and Admission Control in a Cognitive Digital Home
}

\author{
Tianming Li, Student Member, IEEE, Narayan B. Mandayam, Fellow, IEEE, \\ and Alex Reznik, Senior Member, IEEE
}

\begin{abstract}
The advances in radio design and wireless networking, along with the growth of multimedia home entertainment technologies, is creating the concept of a cognitive digital home requiring spectrum coexistence of various devices and networks of networks. A framework for resource allocation in a Cognitive Digital Home (CDH) with a multiplicity of radio access technologies (RAT) such as cognitive radios and legacy radio devices supporting heterogeneous applications is developed. We consider two channel access models in the CDH for addressing spectrum coexistence of legacy devices: (i) Pessimistic Controllability (PC) Model where the Home Genie node (HG) has no influence over legacy devices, and (ii) Switched RAT (SR) Model where the HG has perfect control of legacy devices. Distributed algorithms for maximizing sum rate and maximizing service capacity are designed using partial dual decomposition techniques. A distributed power control scheme is also designed for efficient use of energy. An admission control scheme based on pricing information obtained from the distributed algorithms is used to improve system feasibility.
\end{abstract}

Index Terms-Cognitive digital home, distributed resource allocation, admission control, power control.

\section{INTRODUCTION}

W ITH advances in radio access technologies and the increasing demand on heterogeneous data services, integration of wireless networks with multiple RATs is expected to be a prevalent feature of future mobile networks. Meanwhile, the growth of multimedia services in a home environment for communications, entertainment and safety has also resulted in the concept of a digital home where a multiplicity of devices and RATs coexist. Further, with the advent of cognitive and multi-platform radios, one can envision the spectrum occupancy of these devices and RATs to range from the TV white spaces $(54 \mathrm{MHz} \sim 698 \mathrm{MHz})$ [1], [2] to unlicensed bands $(2.4 \mathrm{GHz}$ and $5 \mathrm{GHz})$ [3] and even all the way to $60 \mathrm{GHz}$ radio bands [4], [5] in a digital home.

The new features of wireless networks in a digital home have drawn some attention from the research community though there are still plenty of interesting research topics to

Manuscript received August 7, 2010; revised December 9, 2011 and January 20, 2012; accepted May 17, 2012. The associate editor coordinating the review of this paper and approving it for publication was N. Arumugam.

T. Li and N. B. Mandayam are with WINLAB, ECE Department, Rutgers University, North Brunswick, NJ 08902 USA (e-mail: \{kevinltm, narayan\}@winlab.rutgers.edu).

A. Reznik is with InterDigital, King of Prussia, PA 19406 USA (e-mail: alex.reznik@interdigital.com).

Part of this paper was presented at the 9th International Symposium on Modeling and Optimization in Mobile, Ad Hoc, and Wireless Networks, Princeton (WiOpt), New Jersey, May 2011.

Digital Object Identifier 10.1109/TWC.2012.011513.111495 be addressed. One of the most important topics in a digital home is the effective operation of multi-RAT networks. There have been some efforts directed at the operation of multiRAT networks in general. In [6], the authors proposed a framework for optimized dynamic usage of radio resources in wireless networks with multi-RATs and multi-operators. Particularly, cognitive radio functionalities have been shown as a must to implement such a system. The authors in [7] studied the network selection process in multi-RAT networks by decomposing it and comparing the common approaches for network selection proposed in literature. In [8], the authors compared the data transmission schemes over multi-RATs with single RAT. They concluded that the algorithm for distributing upper layer data packets over multi-RATs should be carefully designed in order to fully utilize the resources of wireless networks. Radio resource allocation problems in multi-RAT networks were studied in [9] and [10] from the theoretical standpoints. In [9], the authors formulated a utility maximization problem for multi-channel, multi-RAT and multihop wireless networks and a dynamic algorithm was proposed based on the decomposition of this problem. The authors of [10] studied a network throughput maximization problem in a multi-user and multi-RAT network where transmission schemes over multi-RATs and single RAT are both allowed. This problem was shown to be convex and a distributed algorithm based on Dual decomposition was developed to solve it. Besides the effective operation of the networks with existing RATs, integration of new RATs into a digital home is also studied. In [11], the authors began to investigate the feasibility of a new RAT, i.e., cognitive radio systems over TV white space, for a digital home. Analytical and simulation methods have been used to compare the performance of the cognitive radio system with that of license-exempt systems over other spectral bands, e.g., $2.4 \mathrm{GHz}$ and $5 \mathrm{GHz}$ bands.

Although the resource allocation in multi-RAT wireless networks has been studied as discussed above, few efforts [12] [13] have focused on efficient resource allocation in a digital home with multi-RATs. Fair and efficient resource allocation is of paramount importance in supporting various data services. In our earlier work in [12], we have developed a framework for centralized spectrum management in a cognitive digital home $(\mathrm{CDH})$ where a home genie node (HG) coordinates spectrum coexistence across a multiplicity of RATs. Previous works regarding multi-RAT resource allocation usually assume that a RAT and its accessible spectral band are bundled and different RATs' bands don't overlap with each other. However, in a 
$\mathrm{CDH}$, legacy RATs are allowed to coexist and Cognitive Radio (CR) RATs can access all the spectral resources when they are available. These assumptions make our $\mathrm{CDH}$ model different in describing multi-RAT wireless home networks and also add much more complexity to the resource allocation problems within it. For example, the multi-RAT resource allocation problems in [10] can be formulated as a convex optimization and solved by standard methods. However, resource allocation problems in a $\mathrm{CDH}$ are generally $\mathcal{N} \mathcal{P}$-complete and often heuristic algorithms should be carefully designed to trade off the performance and complexity. General analytical models for resource sharing among legacy devices in the same spectral band are difficult to build even though experimental results provide some insights (see [14] and reference therein). In this paper, we consider two models in a $\mathrm{CDH}$ for addressing spectrum coexistence of legacy devices: (i) Pessimistic Controllability (PC) Model, and (ii) Switched RAT (SR) Model. Under the PC model whose preliminary results can be found in [13], we assume that the HG is unable to exercise any control over the devices of legacy RATs and hence cannot influence how the resources are shared by legacy devices that share spectrum. In this case, we assume somewhat pessimistically that each legacy device obtains an equal share of the spectrum resource. Under the SR model, we assume that the HG is able to exercise perfect control over the legacy devices within the parameters of the protocol specifications of each legacy device. Based on the two models, two resource allocation problems (i) Maximizing Sum Rate (MSR), and (ii) Maximizing the Service Capacity (MSC), are formulated. Further, distributed algorithms based on partial dual decomposition are proposed for addressing these problems. Pricing indices play an important role in the design of the distributed algorithms since they convey crucial information regarding a service's achievable and target data rates. A distributed power control scheme based on the channel and RAT allocation result is designed for the services to efficiently use the energy. An admission control scheme based on the pricing indices is also proposed to improve system feasibility when the $\mathrm{CDH}$ system cannot meet all the service requests.

The rest of the paper is organized as follows. The system model of $\mathrm{CDH}$ is presented in section II. In section III, MSR and MSC are formulated. Distributed algorithms, power control scheme and admission control scheme based on partial dual decomposition are designed for them. Extensive numerical results are shown in section IV to evaluate the performance of the system. Section V concludes the paper.

\section{SySTEM Model}

\section{A. Cognitive Digital Home Architecture}

In a $\mathrm{CDH}$, motivated by [15], a spectrum manager, Home Genie Node (HG), coordinates the spectrum coexistence across the home networks, with the degrees of freedom ranging from the transmission parameters such as frequency, bandwidth, power, etc. to the RAT itself. We assume in our model that the $\mathrm{CDH}$ employs devices that support multi-platform radios (MPR) equipped with both CR and legacy RATs. The legacy RATs include technologies such as Bluetooth, Wi-Fi, Zigbee, etc.. The CRs in the $\mathrm{CDH}$ are assumed to be generic

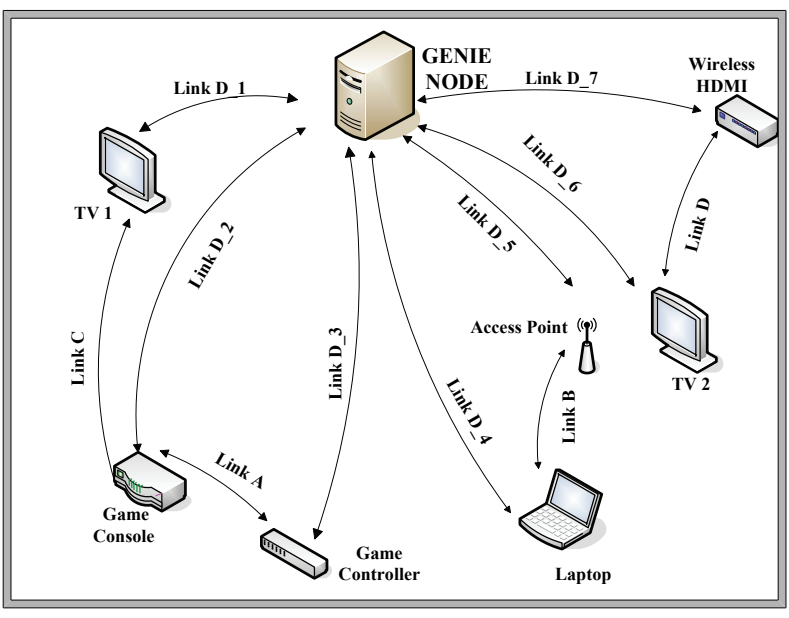

Fig. 1. Illustration of a Cognitive Digital Home

spectrally agile radios capable of noncontiguous Orthogonal Frequency Division Multiple Access (OFDMA) over the entire range of unlicensed frequency spectrum available. The realization of the CR RAT in a CDH relies on advances in hardware and antenna design such as anticipated in future cognitive radio networks [16] [17]. Though a cognitive radio system may allow multiple users to possibly share a channel as in [10], the noncontiguous OFDMA enabled CR RAT [17] considered in our model is designed to support the high rate craving services which usually has high transmit power, e.g., high definition video streaming. Since orthogonal use of a channel maximizes a single service's data rate on that channel, we assume that cognitive radios are opportunistically able to find and use orthogonal channels whenever they are available. Further, we also stipulate that the cognitive radios avoid channels used by legacy RATs so that their performance is not degraded. Parallel transmission [10] where a MPR (service) can simultaneously employ multiple RATs for transmissions is also allowed.

The classes of devices considered in this $\mathrm{CDH}$ model (see Fig. 1) include service provision devices (SPD) which directly provide data services to the end users, e.g., TV and Laptop, as well as relay and wireless access network (RWAN) devices, e.g, Wi-Fi access point and wireless High Definition Multimedia Interface (HDMI), which provide access and relay services to SPDs. All these devices are equipped with MPRs. The HG controls the devices via a set of dedicated control channels as shown in Fig. 1, e.g., link $D_{1}$ for TV 1. The SPDs and RWANs may report their local spectral environment information, data rate requirements or access decisions to $\mathrm{HG}$ via control channels.

\section{B. Preliminaries}

Definition 1. A channel is the atomic unit of spectrum utilized by legacy RATs in a CDH. It is also the atomic unit of spectrum that can be controlled and allocated and, hence, a single orthogonal tone accessed by the noncontiguous OFDMA enabled Cognitive Radio (CR).

The idea of channel allocation is illustrated in Fig. 2, where for simplicity only three channels are shown for each 


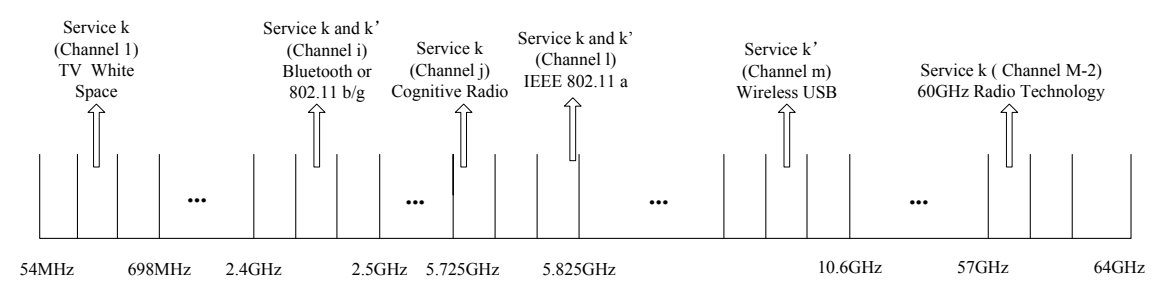

Fig. 2. Exemplary service's channel allocation. Service $k$ and $k^{\prime}$ share channel $i$ and $l$ by using legacy RAT, e.g.,Wi-Fi. Service $k$ exclusively uses channel $j$ by using CR RAT.

portion of spectrum. We assume that the spectrum used by the RATs in the CDH (ISM, TV White Space, Wireless USB and $60 \mathrm{GHz}$ radio) is divided into $M$ equal width channels. However, in the less crowded spectrum bands but with more available resources, e.g., $60 \mathrm{GHz}$ bands, several contiguous channels may be grouped as a single allocation unit for better efficiency. As mentioned above, a service can obtain resources from various RATs and this service may be allocated with noncontiguous channels. Further, in any channel, there could co-exist multiple legacy RATs and multiple services (e.g., see channel $i$ in Fig. 2) for ensuring fairness among services. However, service assigned with CR RAT will exclusively use a channel (e.g., see channel $j$ in Fig. 2) for maximizing the network throughput. This idea can be formally described by a set of channel usage constraints which all the services should comply with,

$$
\begin{aligned}
& x(k, i, t)=\{0,1\}, \forall k \in \mathcal{K}, \forall i \in \mathcal{M}, \forall t \in \mathcal{T} \\
& \mathbf{x}_{\mathbf{k}}=[x(k, 1,1) \ldots x(k, i, 1) \ldots \\
& \quad x(k, i, t) \ldots x(k, i, T) \ldots x(k, M, T)] \\
& \mathbf{l}_{\mathbf{k}}=[l(k, 1,1) \ldots l(k, i, 1) \ldots l(k, i, t) \ldots l(k, i, T) \ldots l(k, M, T)] \\
& 0 \leq l(k, i, t) \leq x(k, i, t), \forall k \in \mathcal{K}, \forall i \in \mathcal{M}, \forall t \in \mathcal{T} \\
& \sum_{k \in \mathcal{K}} \sum_{t \in \mathcal{T}} l(k, i, t) \leq 1, \forall i \in \mathcal{M} \\
& \sum_{t \in \mathcal{T}} x(k, i, t) \leq 1, \forall k \in \mathcal{K}, \forall i \in \mathcal{M}
\end{aligned}
$$

$\mathcal{K}=\{1,2, \ldots, K\}$ and $\mathcal{M}=\{1,2, \ldots, M\}$ are the set of services and set of channels in a $\mathrm{CDH}$, respectively. $\mathcal{T}=\{1,2, \ldots, T\}$ is the set of available RATs on each MPR. $x(k, i, t)$ in (1) indicates whether the $k$-th service uses RAT $t$ in channel $i$ or not. $l(k, i, t)$ in equation (3) shows how the $k$-th service uses technology $t$ to access channel $i$. If the $k$-th service uses CR as its RAT, it has the flexibility to access all the channels in a $\mathrm{CDH}$ and it occupies channel $i$ orthogonally, i.e., $l(k, i, t)=1$, otherwise $l(k, i, t)=0$. If the $k$-th service uses legacy RAT to access channel $i$, the sharing of the channel among services (using legacy RATs) is possible. $l(k, i, t)$ indicates the effective sharing portion that the $k$-th service occupies in channel $i$ and the sharing could occur in the time domain. However, the value of $l(k, i, t)$ is highly dependent on the legacy RATs sharing model employed and we will address this issue below. Different from the CR RAT, legacy RATs can only access a limited number of channels in a specific region, i.e., $\forall i \in \mathcal{M}_{t} \subset \mathcal{M}$, which is predetermined, e.g., IEEE $802.11 \mathrm{~b} / \mathrm{g}$ in $2.4 \mathrm{GHz}$. The total usage of a channel should be no larger than 1 as shown in (5). We assume a service can only employ a single RAT on a channel one time to avoid self-interference as shown in (6).

The coexistence of multiple services with possibly different legacy RATs in the same channel has been studied [18] [19] [20]. However, general analytical models for how resources are shared by legacy devices (e.g., IEEE802.11 and Bluetooth) are not easy to construct. The complexity lies in the interference generated from multiple services with multiple legacy RATs, the differing channels seen by the services and heterogeneous access protocols employed by multiple RATs. As mentioned earlier, in the $\mathrm{CDH}$, we address this complexity via two models (i) Pessimistic Controllability (PC) model, and (ii) Switched RAT (SR) model. Under the PC model, we assume that multiple services assigned to the same channel with possibly different legacy RATs obtain an equal share of the resources. For instance, if $K$ services share a channel, service $k$ with RAT $t$ only achieves $\frac{1}{K}$ of the data rates if service $k$ occupies the channel exclusively with the same RAT $t$. While this is indeed pessimistic, it reflects the reality that the HG is limited in its capability to control the protocol parameters of coexisting legacy RATs. However, in some special cases, the PC model has been shown to be accurate for describing resource sharing among multiple services (as in Carrier Sense Multiple Access (CSMA) networks [21]). Specifically, the PC model is defined by setting:

$$
l(k, i, t)=\left\{\begin{array}{lc}
x(k, i, t) & \text { if } t=\mathrm{CR}, \forall i \in \mathcal{M}, \forall k \in \mathcal{K} \\
\frac{1}{\sum_{k^{\prime} \in \mathcal{K}} \sum_{t^{\prime} \neq \mathrm{CR}} x\left(k^{\prime}, i, t^{\prime}\right)} & \text { if } t \neq \mathrm{CR} \text { and } x\left(k^{\prime}, i, t^{\prime}\right) \neq 0, \\
0 & x(k, i, t) \neq 0, \forall i \in \mathcal{M}_{t}, \forall k \in \mathcal{K} \\
0 & \text { otherwise }
\end{array}\right.
$$

With the advancement of the radio access technologies deployed in a digital home, a better controllability of the HG over the RATs can be expected [22]. Thus, the SR model assumes that the MPRs employs the switched legacy RATs scheme, i.e., only one MPR with a legacy RAT transmits in a channel at a time. The HG may precisely schedule services with legacy RATs to share the channel. Then, $l(k, i, t)$ should take real number value in interval $[0,1]$. The SR model is defined by setting:

$$
l(k, i, t)= \begin{cases}x(k, i, t) & \text { if } t=\mathrm{CR}, \forall i \in \mathcal{M}, \forall k \in \mathcal{K} \\ {[0,1]} & \text { if } t \neq \mathrm{CR}, \forall i \in \mathcal{M}_{t}, \forall k \in \mathcal{K} \\ 0 & \text { otherwise. }\end{cases}
$$


The theoretical physical data rate that can be obtained by the $k$-th service in channel $i$ is given as: $R^{P}(k, i, t)=$ $w_{i} \log \left(1+\frac{h_{k}^{i} P(k, i, t)}{N_{0} w_{i}}\right)$, where $w_{i}$ is the bandwidth of channel $i$. $h_{k}^{i}$ and $P(k, i, t)$ are the $k$-th service's channel gain in the $i$-th channel and transmit power in the $i$-th channel with $t$-th RAT, respectively. $N_{0}$ is the noise level. The effective data rate achieved by the $k$-th service using technology $t$ in channel $i$ is a RAT dependent function, i.e., $R(k, i, t)=f_{t}\left(R^{P}(k, i, t)\right)$. For simplicity, in this paper we assume the relationship is linear and characterized by a factor $\alpha(k, i, t)$, e.g., $R(k, i, t)=$ $\alpha(k, i, t) R^{P}(k, i, t)$, where $0<\alpha(k, i, t) \leq 1$ and $\alpha(k, i, t)=$ $l(k, i, t)$. The data rate achieved by the $k$-th service in channel $i$ is $R(k, i)=\sum_{t \in \mathcal{T}} R(k, i, t)=\sum_{t \in \mathcal{T}} l(k, i, t) R^{P}(k, i, t)$.

\section{Distributed Resource Allocation AND Admission Control in a Cognitive Digital Home}

\section{A. Joint Channel and RAT Allocation Problems}

In the most general setting, the resource allocation in a $\mathrm{CDH}$ includes the assignment to each service a set of channels, corresponding RATs along with choice of transmission power, modulation and coding scheme. In this paper, we focus on Joint Channel and RAT Allocation (JCRA) problems. The first JCRA problem, i.e., Maximizing Sum Rate (MSR), aims to maximize the sum rates while supporting all the inelastic services (rate constrained). By solving this problem, the network efficiency of the $\mathrm{CDH}$ can be maximized while the fairness among services can be guaranteed. We study this problem for both the PC and SR models as follows:

$$
\begin{aligned}
\max _{\mathbf{X}, \mathbf{L}} & \sum_{k \in \mathcal{K}} \sum_{i \in \mathcal{M}} \sum_{t \in \mathcal{T}} R^{P}(k, i, t) l(k, i, t) \\
\text { s.t. } & \sum_{i \in \mathcal{M}} \sum_{t \in \mathcal{T}} R^{P}(k, i, t) l(k, i, t) \geq R_{k}^{\text {min }}, \forall k \in \mathcal{K} \\
& P(k, i, t)=P_{t}, \quad \forall k \in \mathcal{K}, \forall i \in \mathcal{M}, \forall t \in \mathcal{T} \\
& \sum_{i \in \mathcal{M}} \sum_{t \in \mathcal{T}} P(k, i, t) x(k, i, t) \leq P_{k}^{\text {max }}, \forall k \in \mathcal{K}
\end{aligned}
$$

and with (1) - (6) and (7) or (8) for PC or SR, respectively,

where $\mathbf{X}$ and $\mathbf{L}$ are matrices of control variables as defined in (1)-(6). The $k$-th row of $\mathbf{X}$ is $\mathbf{x}_{\mathbf{k}}$ defined in (2) which indicates the channel usage of the $k$-th service over all the channels and RATs. That of $\mathbf{L}$ is $\mathbf{l}_{\mathbf{k}}$ defined in (3) and describes the actual physical resource share of the $k$-th service over all the channels. Note that, under the PC model, $l(k, i, t)$ can be directly calculated from $\mathbf{X}$ as in (7). The control variables are the entries in $\mathbf{X}$ though $l(k, i, t)$ appears in problem formulation and derivations below for necessary conciseness. However, under the SR model, beyond assigning services to a channel with a RAT, the portion of the physical resources it gets should be decided. Constraint (10) requires each service to be satisfied with its own minimal data rate requirement. Constraint (11) reflects the transmit power assumption that it's pre-determined by the RAT itself where a typical value is associated with a RAT as $P_{t}, \forall t \in \mathcal{T}$. This assumption reduces the dimensions of the assignment and makes the problems tractable. Each service (a MPR device) is also limited by its maximal transmit power as shown in (12). Also, the channel usage constraints (1)-(6) cannot be violated and either constraint (7) or (8) is added to it depending on which channel access model is used.

As a special case of the MSR problem (9), Maximizing Service Capacity (MSC) problem is formulated in order to support as many rate constrained services as possible.

$$
\begin{aligned}
\max _{\mathbf{X}, \mathbf{L}} & \sum_{k \in \mathcal{K}} u\left(\sum_{i \in \mathcal{M}} \sum_{t \in \mathcal{T}} R^{P}(k, i, t) l(k, i, t)-R_{k}^{\text {min }}\right) \\
\text { s.t. } & P(k, i, t)=P_{t}, \forall k \in \mathcal{K}, \forall i \in \mathcal{M}, \forall t \in \mathcal{T} \\
& \sum_{i \in \mathcal{M}} \sum_{t \in \mathcal{T}} P(k, i, t) x(k, i, t) \leq P_{k}^{\max }, \forall k \in \mathcal{K}
\end{aligned}
$$

and with (1) - (6) and (7) or (8) for PC or SR, respectively, where $u(x)=1, \forall x \geq 0$ and $u(x)=0, \forall x<0$.

The channel usage constraints (1)-(6) with (7) or (8) add much complexity to the above problems. Depending on which channel access model is used, i.e., PC model or SR model, the problems are named as PC-MSR, PC-MSC, SR-MSR and SRMSC. The hardness of the channel usage constraints lies in the assumptions that the CR RAT can access all the channels and multiple services can access the same spectral resources with multiple RATs. These assumptions capture the features of $\mathrm{CDH}$ as envisioned in the future along with advances in cognitive radio technology. Thus, these problems are worthwhile to solve regardless of their hardness. Before proceeding to algorithm design, the complexities of the problems are first studied.

Theorem 1. The PC-MSR, PC-MSC, SR-MSR and SR-MSC are all $\mathcal{N} \mathcal{P}$-complete.

Proof: In the first place, PC-MSR can be shown to be $\mathcal{N} \mathcal{P}$. The decision problem of PC-MSR can be described as "given a real number $P \in \mathbb{R}_{+}$, can the solution to PC-MSR problem (9) support sum rates such as $\sum_{k \in \mathcal{K}} \sum_{i \in \mathcal{M}} \sum_{t \in \mathcal{T}} R(k, i, t) l(k, i, t) \geq P$ ? ". Given a solution, i.e., $\left\{\mathbf{X}^{*}, \mathbf{L}^{*}\right\}$, the correctness of this solution can be verified efficiently, i.e., in polynomial time. We assume that the calculation time of $R^{P}(k, i, t)$ is constant, then the total time to calculate the sum rate and verify the individual rate requirements can be bounded by $\mathcal{O}(|\mathcal{K}\|\mathcal{M}\| \mathcal{T}|)$. Therefore, we can show PC-MSR $\in \mathcal{N} \mathcal{P}$.

Next, we examine the PC-MSR problem from a different angle and polynomially reduce a 2-dimensional Multiple Knapsack Problem (MKP) [23] [24] to it. By doing this, we first consider a Simpler variation of PC-MSR (SPC-MSR) where we assume only legacy RAT $t^{\prime}$ is used and $R\left(k, i, t^{\prime}\right)$ is predetermined. Then, we may consider that each service has its own knapsack constrained by its own maximal transmit power $P_{k}^{\max }$ and its minimal data rate $R_{k}^{\text {min }}$. For each channel $i$, there are $|\mathcal{K}|$ objects affiliated to it, e.g., the $k$ th service with RAT $t^{\prime}$ on channel $i$. This object has constant transmit power size $P_{t^{\prime}}$ and transmit rate value $R\left(k, i, t^{\prime}\right)$. Then, the SPC-MSR problem aims to maximize the sum transmit rate value of a subset of the $|\mathcal{K}||\mathcal{M}|$ objects while the objects are feasibly packed into the $|\mathcal{K}|$ knapsacks. Thus, the correspondence from a 2-dimensional multiple knapsack problem to SPC-MSR problem is established and we have shown that MKP can be polynomially reduced to SPC-MSR problem, i.e., MKP $\preceq_{p}$ SPC-MSR. With the facts that MKP 
is strongly $\mathcal{N} \mathcal{P}$-complete, PC-MSR $\in \mathcal{N P}$ and PC-MSR is harder than SPC-MSR, we can prove that PC-MSR is also strongly $\mathcal{N} \mathcal{P}$-complete. The strongly $\mathcal{N} \mathcal{P}$-completeness of PC-MSC, SR-MSR and SR-MSC problems can be proven in the same manner. PC-MSR (PC-MSC) problem is harder than SR-MSR (SR-MSC) problem since it includes nonlinear constraints.

From Theorem 1, none of the four problems is shown to have an efficient algorithm. Thus, sensible heuristic algorithms are preferred to balance the system performance and computational complexity. Further, distributed algorithms are preferred in order to reduce the computation and sensing burden at the HG. Therefore, we will design distributed algorithms for JCRA based on partial dual decomposition as discussed below.

\section{B. Distributed Algorithms for PC-MSR and PC-MSC Prob- lems}

Note that in the PC-MSR problem (9), the minimal rate requirements (10) and maximal transmit power requirements (12) are all service-wise. Further, the RAT power constraint (11) does not imply any coupling among the services. Thus, an intuitive idea is to decompose this problem service-wise by relaxing either (10) or (12) or both into the objective function [25] with the only constraint implying couplings among services being the channel usage constraints (1)-(6) and (7). For the PC-MSR problem, the major concern is to make the system feasible and thus relaxing individual rate requirements into the objective function is preferred. The partial dual function can be obtained by solving the following problem:

$$
\begin{array}{r}
\max _{\{\mathbf{X}, \mathbf{L}\} \in \mathcal{Q}} \mathcal{L}(\mathbf{X}, \underline{\lambda})=\sum_{k \in \mathcal{K}} \sum_{i \in \mathcal{M}} \sum_{t \in \mathcal{T}} R^{P}(k, i, t) l(k, i, t) \\
+\sum_{k \in \mathcal{K}} \lambda_{k}\left(\sum_{i \in \mathcal{M}} \sum_{t \in \mathcal{T}} R^{P}(k, i, t) l(k, i, t)-R_{k}^{\text {min }}\right) \\
\text { s.t. } P(k, i, t)=P_{t}, \quad \forall k \in \mathcal{K}, \forall i \in \mathcal{M}, \forall t \in \mathcal{T} \\
\sum_{i \in \mathcal{M}} \sum_{t \in \mathcal{T}} P(k, i, t) x(k, i, t) \leq P_{k}^{\text {max }}, \forall k \in \mathcal{K}
\end{array}
$$

and with channel usage constraints (1) - (6) and (7),

where $\mathcal{Q}$ is the feasible set defined by (17), (18) and constraints (1)-(6) and (7). $\lambda_{k} \geq 0, \forall k \in \mathcal{K}$ is the Lagrangian multiplier and $\mathcal{L}(\mathbf{X}, \underline{\lambda})$ is the Lagrangian function. Assume that $\left\{\mathbf{X}^{*}, \mathbf{L}^{*}\right\}$ is the optimal solution to problem (16), then the dual problem (master problem) can be defined as:

$$
\begin{aligned}
& \min _{\underline{\underline{\lambda}} \in \mathbb{R}_{+}^{K}} \mathcal{L}(\underline{\lambda})=\sum_{k \in \mathcal{K}} \sum_{i \in \mathcal{M}} \sum_{t \in \mathcal{T}} R^{P}(k, i, t) l^{*}(k, i, t) \\
& \quad+\sum_{k \in \mathcal{K}} \lambda_{k}\left(\sum_{i \in \mathcal{M}} \sum_{t \in \mathcal{T}} R^{P}(k, i, t) l^{*}(k, i, t)-R_{k}^{\text {min }}\right),
\end{aligned}
$$

where $\underline{\lambda}=\left[\lambda_{1} \ldots \lambda_{k} \ldots \lambda_{K}\right]$ is the Lagrangian multiplier vector and $l^{*}(k, i, t)$ can be computed directly from $\mathbf{X}^{*}$. If strong duality holds for the problem (9) and its dual problem (19), the optimal solution to (9) can be identified from the feasible solutions of (19) and additional conditions [26]. Unfortunately, problem (16) is still $\mathcal{N} \mathcal{P}$-hard and furthermore no strong duality relation can be proven here. Thus, an optimal solution cannot be identified by following the standard dual decom- position methods. However, this partial dual decomposition structure is still helpful for developing heuristic distributed algorithms. Problem (16) can be decomposed into $K$ local sub-problems and as for the $k$-th service:

$$
\begin{aligned}
\max _{\left\{\mathbf{x}_{\mathbf{k}}, \mathbf{l}_{\mathbf{k}}\right\}} & \sum_{i \in \mathcal{M}} \sum_{t \in \mathcal{T}} R^{P}(k, i, t) l(k, i, t) \\
& +\lambda_{k}\left(\sum_{i \in \mathcal{M}} \sum_{t \in \mathcal{T}} R^{P}(k, i, t) l(k, i, t)-R_{k}^{\text {min }}\right) \\
\text { s.t. } \quad & P(k, i, t)=P_{t}, \quad \forall i \in \mathcal{M}, \forall t \in \mathcal{T} \\
& \sum_{i \in \mathcal{M}} \sum_{t \in \mathcal{T}} P(k, i, t) x(k, i, t) \leq P_{k}^{\max }
\end{aligned}
$$

and with channel usage constraints (1) - (6) and (7),

where the $k$-th service here can only determine its own channel access strategy, i.e., deciding its channel access indicator vector $\mathbf{x}_{\mathbf{k}}$. Meanwhile, the potential violation of the channel usage constraints (1) - (6) and (7) can be avoided with the help of the HG. Though the HG doesn't make centralized spectrum allocation decisions, it can obtain channel usage information from all the services and maintain them in a Global Spectrum Map (GSM). Then, the HG can pass the channel usage information of the other services from the GSM to the service $k$. The $k$-th service can therefore avoid collisions and calculate its own achievable data rates. A modified greedy algorithm is used here by the $k$-th service. The general idea of the modified greedy algorithm is that the individual service should maximize its data rate when global resources are sufficient. However, when the global resources are scarce, the individual service should aim to achieve its rate requirement while allowing possible sharing of the physical resources for other services. As mentioned above, GSM is only used as a simplified method to meet the channel usage constraints (1) - (6) and (7). It avoids exponentially enumerating all possible channel and RAT allocations of all the services but meanwhile introduces performance degradation to the proposed distributed algorithms. If strong duality holds for the primal and dual problems, i.e., (9) and (19), then the optimal solution can be obtained through standard method [26], i.e., iterative improvement of feasible primal and dual solutions. However, the $\mathcal{N} \mathcal{P}$-hardness of primal, dual and local problems prevents us from deriving such results. The solution, i.e., $\left\{\mathbf{X}^{\prime}, \mathbf{L}^{\prime}\right\}$, from the $K$ local sub-problems via the local modified greedy algorithm, may not even be feasible. Thus, we need to improve the current solution to make it feasible. We propose a pricing index, i.e., $P I_{k}, k \in \mathcal{K}$, to indicate the service $k$ 's priority to obtain resource assignment to meet its data rate requirement. A larger value of $P I_{k}$ represents that service $k$ has a larger resource insufficiency. If the current allocation is infeasible, the pricing index is updated as:

$$
\begin{aligned}
& P I_{k}(i t r+1)= \\
& {\left[P I_{k}(i t r)-\alpha(i t r)\left(\sum_{i \in \mathcal{M}} \sum_{t \in \mathcal{T}} R^{P}(k, i, t) l^{\prime}(k, i, t)-R_{k}^{m i n}\right)\right]^{+}}
\end{aligned}
$$

where $i t r$ is the iteration index and $\alpha(i t r)>0$ is the step size in iteration $i t r .[\cdot]^{+}$denotes the projection onto the nonnegative orthant. The current pricing index is calculated based on both the services' achievable data rates in the current alloca- 


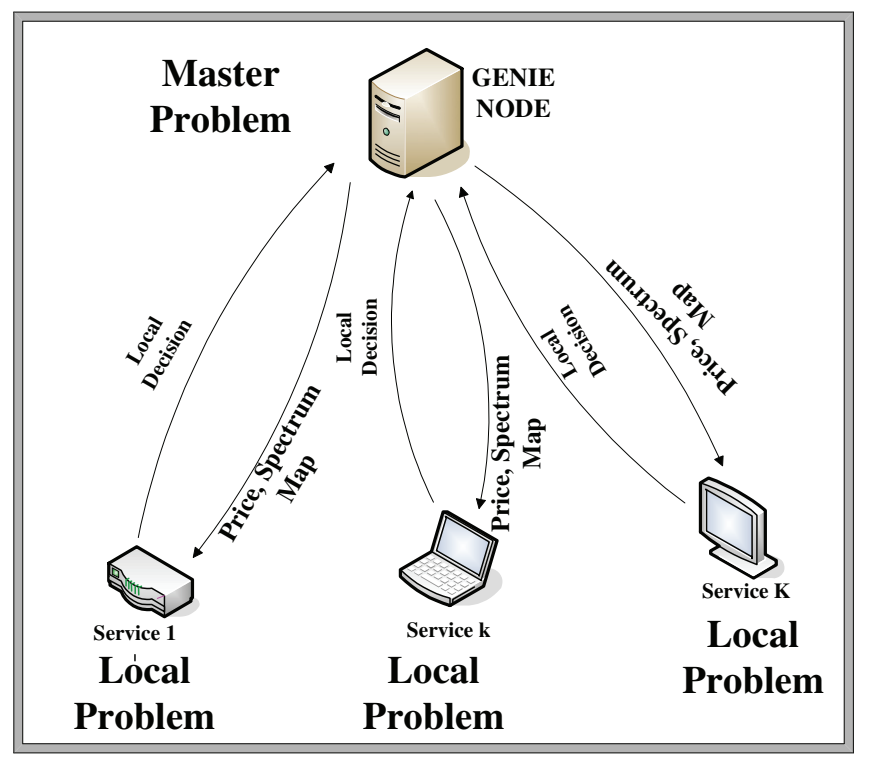

Fig. 3. Decomposition of Resource Allocation Problems. PC-MSR problem can be decomposed as a master problem and $|\mathcal{K}|$ local problems. The sensing and part of computation burden is distributed to local services. The HG only needs to maintain and update the global spectrum information.

tion, i.e, $\left\{\mathbf{X}^{\prime}, \mathbf{L}^{\prime}\right\}$, and their pricing index in the last infeasible resource claim iteration. If the $k$-th service is not satisfied with its data rate requirement in the current resource claim iteration, its pricing index $P I_{k}$ will be increased according to (23) in its next resource claim iteration $i t r+1$. Otherwise, if the $k$-th service has been assigned much redundant resources, its $P I_{k}$ will be decreased. Then, the services will claim resources in the descending order of their pricing indices. The pricing index update in (23) should not be considered the same as the Lagrangian multipliers update in standard dual decomposition method [25]. Here, it only determines the order of the services to claim resources in the next iteration. A threshold for the number of resource claim iterations, i.e., MaxItr, must be set in implementation and its impact is discussed in the numerical results section. If a feasible allocation cannot be found within the threshold, the system is claimed to be infeasible. The partial dual decomposition is used as a guideline to decompose the PC-MSR problem (9) as in Fig. 3 rather than exactly solve it. The HG only needs to update the pricing indices and maintains GSM. The sensing burden and part of the computation are distributed to local services. Based on this decomposition, a 3-stage Distributed algorithm for the PCMSR that we refer to as D-PCM can be developed as in Fig. 4. Since the first two stages of the distributed algorithm aims to support as many rate constrained services as possible, the PC-MSC problem can also be solved with this algorithm. The D-PCM algorithm can be explained in detail as follows:

(i) Priority Initialization Initial values for pricing indices are computed in this stage. The initial value of pricing index reveals the gap between service's potential achievable data rates and its minimal data rate requirement. The $k$ th service's potential achievable data rates can be estimated by its average achievable data rates across all the channels, i.e., $R_{k}^{E}=\frac{\sum_{i \in \mathcal{M}} w_{i} \log \left(1+\frac{h_{k}^{i} P_{k}^{\max }}{N_{0} w_{i}}\right)}{M}$. Then, its target rates to potential rates ratio is defined as $T P_{k}=\frac{R_{k}^{m i n}}{R_{k}^{E}}$. Pricing indices are initialized as $P I_{k}(0)=T P_{k}, \forall k \in \mathcal{K}$ and all the services are sorted in the decreasing order of the pricing indices. With a larger pricing index, the service has higher probability of not achieving minimal data rate requirement and thus should be assigned a higher resource claim priority. The HG disseminates the pricing indices to all the services and the services claim the resources in the order mentioned above.

(ii) Min. Rate Allocation All the MPR devices in a $\mathrm{CDH}$ are assumed to be able to obtain their own channel gains and the HG node can disseminate the updated channel usage information (GSM) to them through the control channels. The channel usage information records the services which transmit in each channel and the corresponding RATs employed. Based on this information, the services can solve their local problem (20). The service with the highest pricing index gets the highest priority to solve the local problem and claims the resources from the HG. The other services solve their own local problems and claim the resources from the $\mathrm{HG}$ in the order of decreasing initial pricing indices.

For the $k$-th service, its local problem aims to maximize its data rate to satisfy the minimal data rate requirement subject to the transmit power and channel usage constraints. Meanwhile, the $k$-th service should also consider the other services' minimal data rate requirements and not demand too much resources. Hence, depending on the relationship between the number of services $|\mathcal{K}|$ and that of channels $|\mathcal{M}|$, the service can use a different strategy to solve its local problem. The $k$-th service starts from its best channel (the highest channel gain) and searches over all the available channels in the order of decreasing channel gains. On the channel $c$, if $|\mathcal{M}|<|\mathcal{K}|$, the $k$-th service starts from the lowest transmit power RAT and searches over all the RATs in the order of increasing transmit power. On the contrary, if $|\mathcal{M}| \geq|\mathcal{K}|$, the $k$-th services searches the RATs in the decreasing order of the transmit power. Given that the $k$-th service can achieve $R_{k}=\sum_{i=1}^{c-1} \sum_{t \in \mathcal{T}} R^{P}(k, i, t) l(k, i, t)$, if $R_{k}+R^{P}(k, c, t) l(k, c, t) \geq R_{k}^{m i n}$ and this possible allocation does not violate the channel usage constraints (1)-(6) and (7), the $k$-th service will claim this resource usage from the HG. Otherwise, it will check the $(t+1)$-th RAT that has next higher (or lower) transmit power. If $t=T$ and $R_{k}+R^{P}(k, c, t) l(k, c, t)<R_{k}^{\text {min }}$ that means no RATs in the channel $c$ can provide the sufficient additional rates to satisfy the service's minimal data rate requirement, the $k$-th service will claim $R_{k}=R_{k}+R^{P}\left(k, c, t^{\prime}\right) l\left(k, c, t^{\prime}\right)$ where $t^{\prime} \in \mathcal{T}$ is the feasible RAT with the largest transmit power for the $k$-th service in the $c$-th channel and then go to next channel $c+1$ with next lower channel gain. This search process continues until the $k$-th service goes through all its accessible channels and sends its channel and RATs usage claim and achievable data rate to the HG.

After the HG gets the $k$-th service's resource usage claim, it updates the GSM and sends it to the next service in the priority list to claim the resources. When the $\mathrm{HG}$ gets the resource usage claims from all the $|\mathcal{K}|$ services, it can calculate the gaps between each service's achievable and minimal required 


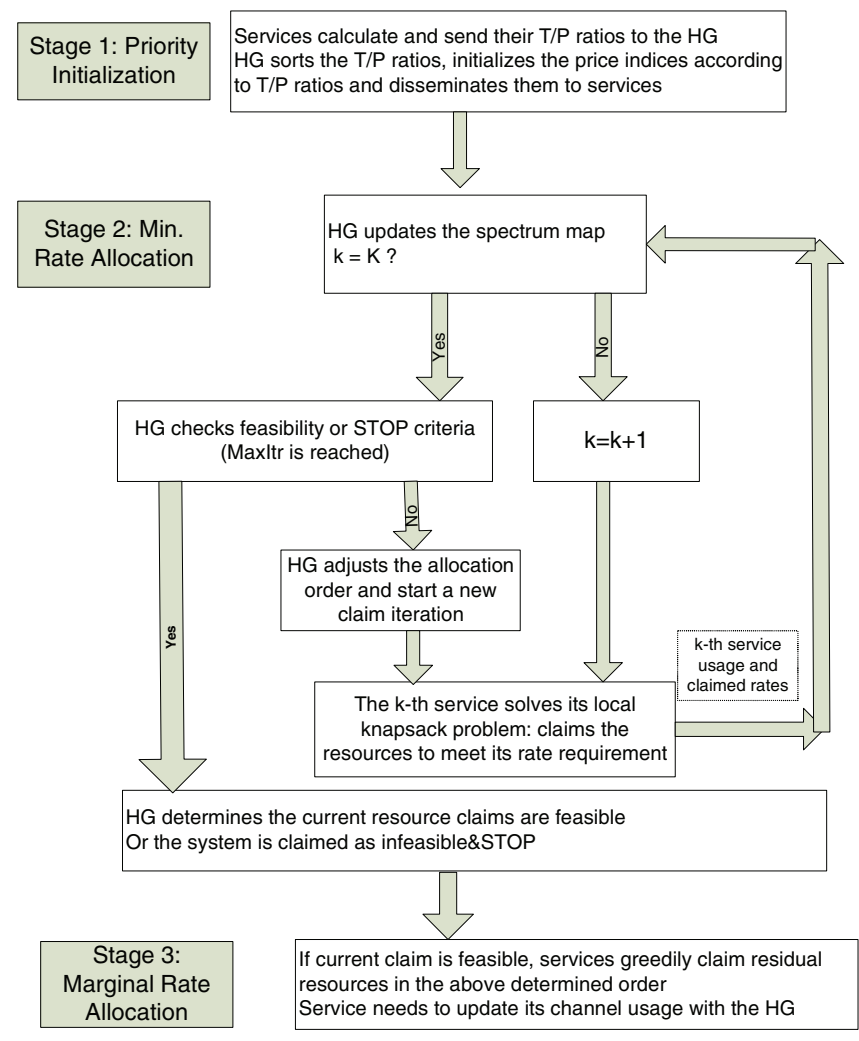

Fig. 4. D-PCM: Distributed Algorithm for PC-MSR/MSC. The algorithm is designed based on partial dual decomposition. Pricing indices are used to indicate the gap between a service's achievable rate and its target rate. It's also an important module of D-SRM, the distributed algorithm for SR-MSR/MSC.

rates, i.e., $\sum_{i \in \mathcal{M}} \sum_{t \in \mathcal{T}} R^{P}(k, i, t) l(k, i, t)-R_{k}^{\min }$. If all the services achieve their rate requirements, the HG sends the permissions to all the services for their feasible claims. If some service cannot be satisfied with its rate requirement, the HG updates the pricing indices according to (23) and sorts them in the decreasing order that is also the new order for the services to claim the resources. The same procedures of local problem solving, resource usage claim and the pricing indices updates will be executed until the system is feasible or the number of resource claim iterations exceeds a pre-defined threshold MaxItr.

(iii) Marginal Rate Allocation If the resource allocation in the stage 2 can satisfy all the services' rate requirements, the services will access the residual spectrum resources in this stage. The services access the residual resources in the order that is finalized in stage 2. For each service, it accesses the channel and RAT pair which is available and provides it the largest additional data rate. One important rule of access is that services will not access the channels which are already shared by other services with legacy RATs in stage 2 since the potential sharing will possibly undermine the feasibility of the system.

The stage 1 of D-PCM requires information of $|\mathcal{K}|$ services over $|\mathcal{M}|$ channels to initialize the pricing indices which results in $\mathcal{O}(|\mathcal{K}||\mathcal{M}|)$ complexity. In stage 2 , each service $k \in \mathcal{K}$ needs to check each RAT and channel pair in each iteration. Since the number of the resource claim iterations is chosen to be a small constant, the complexity in stage 2 is $\mathcal{O}(|\mathcal{K}||\mathcal{M}||\mathcal{T}|)$. Stage 3 only requires $\mathcal{O}(|\mathcal{K} \| \mathcal{M}|)$. In total, the D-PCM is of complexity $\mathcal{O}(|\mathcal{K}||\mathcal{M} \| \mathcal{T}|)$ which shows it is a fast algorithm with polynomial time complexity.

\section{Distributed Algorithms for SR-MSR and SR-MSC Prob- lems}

As proven in section III, the PC-MSR, PC-MSC, SR-MSR and SR-MSC problems are all strongly $\mathcal{N} \mathcal{P}$-complete which entail forbidden complexity to find their optimal solutions. The SR-MSR and SR-MSC problems can be readily solved by the branch and bound algorithm [23] which is of asymptotically exponential complexity and centralized. From (7) and (8), we can identify that the feasible sets of the PC-MSR and PCMSC are proper subsets of that of the SR-MSR and SRMSC, respectively. Thus, the optimal solution of SR-MSR (SR-MSC) problem gives an upper bound for that of PCMSR (PC-MSC) problem and can be used as a benchmark to evaluate the performance of D-PCM. As in case of PC-MSR and PC-MSC problems, a two stage Distributed algorithm for the SR-MSR and SR-MSC problems that we refer to as DSRM is designed as follows.

In the first stage of the D-SRM, an initial channel and RAT allocation should be obtained in a distributed manner. The D-PCM designed in Fig. 4 has two attractive features: (i)D-PCM will output an initial channel and RAT allocation efficiently, and (ii)D-PCM is distributed where the majority of the computation and sensing burden is assigned to the services. Thus, the D-PCM becomes a proper subroutine candidate here. In the second stage, with the initial channel and RAT allocation, i.e., $\widetilde{\mathbf{X}}$, the HG solves a simple linear program (LP) to refine the resource allocation on the channels with legacy RATs to determine the exact portion of the physical resources each service will obtain. The legacy RATs refinement LP can be formulated as:

$$
\begin{aligned}
\max _{\mathbf{L}} & \sum_{k \in \mathcal{K}} \sum_{i \in \mathcal{M}} \sum_{t \neq \mathrm{CR}} R^{P}(k, i, t) l(k, i, t) \\
\text { s.t. } & \sum_{i \in \mathcal{M}} \sum_{t \neq \mathrm{CR}} R^{P}(k, i, t) l(k, i, t) \geq R_{k}^{r e s}, \quad \forall k \in \mathcal{K} \\
& \sum_{k \in \mathcal{K}} \sum_{t \neq \mathrm{CR}} l(k, i, t)=1, \quad \forall i \in \mathcal{M} \\
& 0 \leq l(k, i, t) \leq \widetilde{x}(k, i, t), \quad \forall k \in \mathcal{K}, \quad \forall i \in \mathcal{M},
\end{aligned}
$$

where $R_{k}^{r e s}=R_{k}^{\text {min }}-\sum_{i \in \mathcal{M}} \sum_{t=\mathrm{CR}} R^{P}(k, i, t) l(k, i, t)$ is the residual part of the rate constraint which should be satisfied with legacy RATs and part of the rate constraint may have been satisfied by CR RAT in stage 1 . The initial allocation $\widetilde{\mathbf{X}}$ results in an feasible allocation. Thus, no power constraint is required here. Meanwhile, only the services assigned with legacy RAT on a channel in $\widetilde{\mathbf{X}}$ can be further considered for allocation on that channel here as in (27). The above LP can be efficiently solved by the HG since it readily obtains the necessary information to solve the LP in the first stage and the problem size is usually small in a digital home.

\section{Power Control in a Cognitive Digital Home}

Besides channel and RAT allocation discussed above, power control is another important issue for managing resources in a 
$\mathrm{CDH}$. However, as we have mentioned in Theorem 1, the joint channel and RAT allocation itself is already $\mathcal{N} \mathcal{P}$-complete. Adding power control as an additional degree of control, i.e., joint channel, RAT and power allocation, will make the problem even more intractable. To overcome the intractability, we propose power control in a $\mathrm{CDH}$ as an additional functionality which can improve the system performance based on the allocation results from D-PCM or D-SRM algorithms. Given the channel and RAT allocation result obtained from D-PCM or D-SRM, i.e, $\mathbf{X}^{*}$ and $\mathbf{L}^{*}$, the power control can be implemented locally by each service as follows:

$$
\begin{aligned}
\max _{\mathbf{p}_{\mathbf{k}}} & \sum_{i \in \mathcal{M}} \sum_{t \in \mathcal{T}} R^{P}(k, i, t) l^{*}(k, i, t) \\
\text { s.t. } & \sum_{i \in \mathcal{M}} \sum_{t \in \mathcal{T}} P(k, i, t) x^{*}(k, i, t) \leq P_{k}^{\max } \\
& 0 \leq P(k, i, t) \leq P_{t}^{\text {max }}, \forall i \in \mathcal{M}, \forall t \in \mathcal{T},
\end{aligned}
$$

where each service tries to locally maximize its achieved data rates given the channel and RAT assignment. As in equation (11), the joint channel and RAT assignment is based on some fixed nominal transmit power value $P_{t}$. Subsequently, each service adjusts its transmission power $P(k, i, t)$ for each channel and RAT here, corresponding to the constraints (29) and (30), respectively. For fixed constraints $P_{k}^{\max }$ (service) and $P_{t}^{\max }$ (RAT), the above problem (28) is a convex program and thus can be solved efficiently. Moreover, in a typical CDH environment, given the relatively short range of communications, the dynamic range of power control will typically be less than in a macrocell setting.

\section{E. Admission Control in a Cognitive Digital Home}

As emphasized in previous sections, the JCRA problems, e.g., PC-MSR and SR-MSR, are all $\mathcal{N} \mathcal{P}$-hard and thus their feasibility is hard to achieve. This is the reason why the D-PCM and D-SRM algorithms weigh more importance on the feasibility of the problems besides the total sum rates. The infeasibility of the systems when the physical resources are limited requires the $\mathrm{CDH}$ system to employ an admission control scheme. From the system aspect, the admission control scheme should help the HG efficiently determine which services should be accepted for data services. From the optimization aspect, the admission control scheme should serve as a feasibility seeking algorithm to reduce the constraint set size so that the reduced optimization problem becomes feasible.

In general, seeking feasibility of an optimization problem can be formulated as the minimum-cardinality IIS (Irreducible Infeasible Set) set-covering problem that is known to be $\mathcal{N} \mathcal{P}$-hard [27]. Heuristic methods such as in [28] [29] have been developed for it, but the current state of the art mainly targets linear systems [27]. The JCRA problems in a CDH are in general nonlinear. Therefore, specific admission control methods should be developed to address the infeasibility issue. The pricing indices $P I_{k}$ in (23) used in resource allocation provide a nice indication for admission control since they represent the gap between each service's target data rate and its achievable data rate in the resource claim iteration. One example of a service rejection scheme based on the

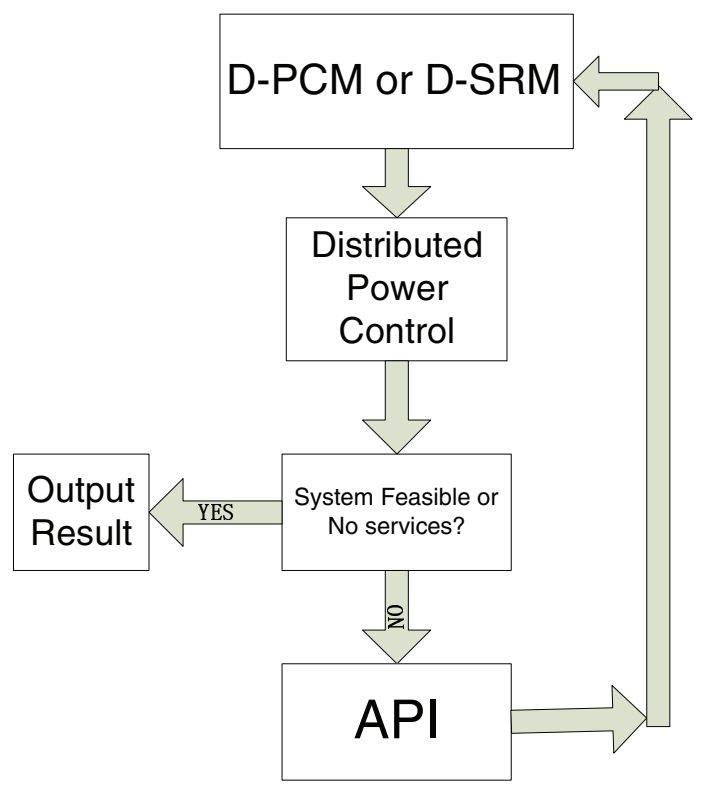

Fig. 5. Distributed Power Control Scheme and Admission Control Scheme(API). D-PCM and D-SRM are the distributed algorithms for PCMSR(MSC) and SR-MSR(MSC), respectively. Power control is completed locally by each service based on a feasible joint channel and RAT assignment. The pricing index reveals the gap between a service's achievable rates and target rates and thus is a proper indication on the service rejection candidate.

pricing indices first we consider is the Average Pricing Index (API) scheme. The API scheme rejects the service with the largest average pricing index, i.e., $k=\arg \max _{i \in \mathcal{K}} \overline{P I_{i}}=$ $\arg \max _{i \in \mathcal{K}} \frac{\sum_{i t r=1}^{M a x} P I_{i}(i t r)}{\text { MaxItr }}$, where MaxItr is the maximum number of resource claim iterations before the system is determined infeasible. The API scheme will reject the services until the remaining system is determined feasible by either D-PCM or D-SRM or all the services are rejected. The API scheme can be appended to the end of the D-PCM or D-SRM algorithms as shown in Fig. 5 where the power control block is also shown. The performance of the API scheme will be discussed next.

\section{Numerical Results}

We first illustrate our results in a simple setting that exemplifies a CDH environment with Wi-Fi, bluetooth and cognitive RATs which use fixed transmit power on each channel for each service. The spectral environment parameters used in the MATLAB-based simulation are shown in Table 1. Due to the directional nature of the wireless links in $60 \mathrm{GHz}$ bands, the link gains of each service in $60 \mathrm{GHz}$ bands can be described by a static path loss channel model as $\frac{P_{r}}{P_{t}}=\left(\frac{c}{4 \pi d_{0} f}\right)^{2} \times\left(\frac{d_{0}}{d}\right)^{\gamma}$, where $P_{r}$ and $P_{t}$ are received and transmit powers. $d$ is the link distance between the transmitter and receiver. $d_{0}=1 \mathrm{~m}$ is the indoor reference distance. $f$ and $c$ are the channel center frequency and speed of light, respectively. $\gamma$ is the indoor path loss coefficient that is chosen to be $\gamma=1.8$ for line of sight (LOS) links in $60 \mathrm{GHz}$ bands [30] [31]. For the $2.4 \mathrm{GHz}$ and $5 \mathrm{GHz}$ bands, the Rayleigh fading model is used to calculate the link gains of each service as $\frac{P_{r}}{P_{t}}=\left(\frac{c}{4 \pi d_{0} f}\right)^{2} \times\left(\frac{d_{0}}{d}\right)^{\gamma} \times X_{g}$, where the path loss coefficient is chosen to be $\gamma=3$ [32] and $X_{g}$ is a random variable with Rayleigh distribution with zero mean and unit variance. 
The first numerical simulation is for an exemplary scenario where a $\mathrm{CDH}$ has service provision devices such as a game console, game controller, laptop and High Definition TV (HDTV) as shown in Fig.1. The link A (game controller) is assumed to be a legacy bluetooth link at $1 \mathrm{Mbps}$ and we consider channel and RAT allocation for the link B (web surfing), C (game display) and D (wireless HDTV). The available channels and their bandwidths are shown in Table I. We assume Wi-Fi and bluetooth can operate only on the first 4 channels in the $2.4 \mathrm{GHz}$ band while the CRs can operate on all available channels. The degradation of Wi-Fi due to bluetooth is modeled via a degradation factor $\alpha_{t}=0.9$ [18]. The allocation results also shown in Table I verify that our D-PCM algorithm efficiently utilizes the integrated spectrum and RATs resources in the CDH. The services with high data rate requirements which usually also have high transmit power margin, e.g., the wireless HDTV, are pushed to higher frequency bands with CR RAT. The services with low transmit power margin and moderate rate demands, e.g., web surfing, are allocated to the ISM bands with Wi-Fi legacy RATs. The sharing nature of some legacy RATs such as WiFi can accommodate more such services to meet their data rate requirements than the orthogonalized CR RAT while CR RAT can provide more flexibility to support high rate craving services.

In the following, we will investigate the performance of DPCM and D-SRM algorithms. The performance of D-PCM algorithm will be compared with the upper bound of its optimal solution generated by the SR-MSR problem. It will also be compared with that of the D-SRM algorithm to study the gain due to the relaxation of the legacy RATs access. The channels of $20 \mathrm{MHz}$ wide each which are centered at 5.18 , $5.2,5.22,5.24,5.26,5.28,5.3,5.32,5.745,5.765,5.785$ and $5.805 \mathrm{GHz}$ are used here. In the first place, we will investigate the performance of D-PCM and D-SRM as the function of the number of services $|\mathcal{K}|$ and the number of channels $|\mathcal{M}|$. Two metrics, i.e., the sum rates of all the services and the system feasibility rate, are compared in the following figures. If all the services' rate requirements are satisfied, the system is claimed to be feasible. The sum rate is defined as the sum of all the services' rates if the system is feasible. Here, all the services are of the same rate requirements, i.e., $R_{k}^{\min }=10 \mathrm{Mbps}$ for each $k \in \mathcal{K}$. Their link distances are uniformly generated over $[5,10] \mathrm{m}$. The services also have the same individual transmit power constraint, i.e., $P_{k}^{\max }=200 \mathrm{~mW}$ for $\forall k \in \mathcal{K}$. Two RATs, i.e., Wi-Fi with typical transmit power $30 \mathrm{~mW}$ and CR with typical transmit power $60 \mathrm{~mW}$, are employed in the $\mathrm{CDH}$ where Wi-Fi RAT is allowed in the first 3 channels and the CR RAT can possibly be used in all the channels. For each choice of number of services and number of channels, the results (the actual allocation for each of 100 trials is not shown) shown are averaged over 100 trials.

In Fig. 6, the performance of D-PCM in terms of the system feasibility rate is compared with that of D-SRM. The system feasibility rate is defined as the percentage of the feasible trials out of the total 100 trials. The D-PCM algorithm almost obtains the optimal solution in Fig. 6. As the number of services increases, its performance will degrade as expected. The D-SRM algorithm which is based on D-PCM algorithm

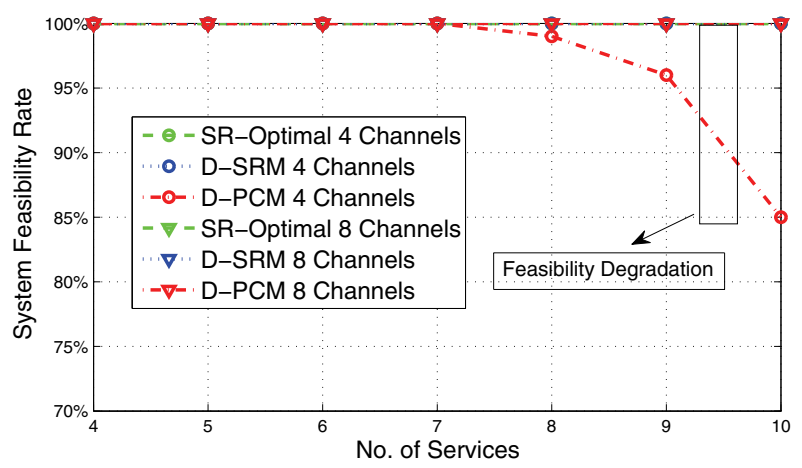

Fig. 6. System Feasibility Rate. The services have same individual rate requirement of $10 \mathrm{Mbps}$.

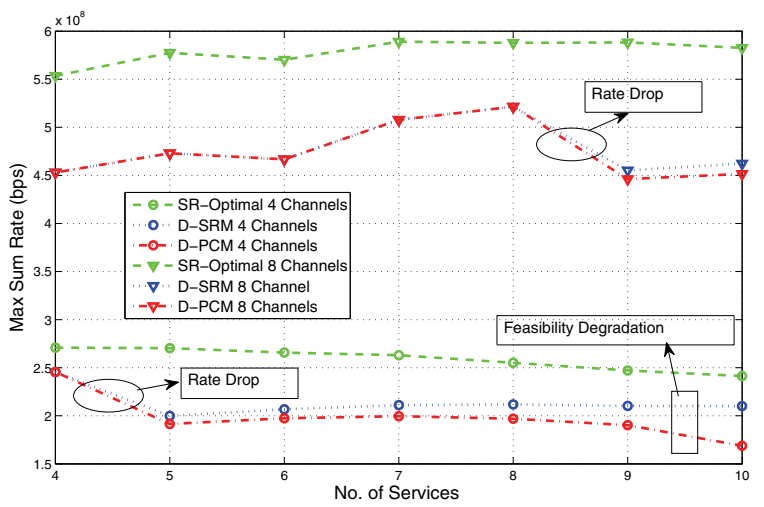

Fig. 7. Max Sum Rate. The services have same individual rate requirement of $10 \mathrm{Mbps}$.

obtains the optimal solution in this case. The system feasibility degradation of D-PCM shown in the figure actually reflects the limited controllability of legacy RATs in the PC-MSR problem.

The performance of D-PCM and D-SRM in terms of the sum rate are compared in Fig. 7. Both D-PCM and D-SRM have less than $25 \%$ performance degradation relative to the optimal solution of the SR-MSR problem. This also indicates that the gap between the performance of D-PCM and the optimal solution of PC-MSR is lower than $25 \%$. As shown in the figure, when $|\mathcal{K}|>|\mathcal{M}|$, there is a sum rate drop for both DPCM and D-SRM. This is due to the RAT access strategy used in the two algorithms. As mentioned above, when $|\mathcal{K}| \leq|\mathcal{M}|$, both the algorithms push the services to aggressively access the RATs to maximize their rates. However, when the physical resource is scarce, the algorithms encourage the services to conservatively share spectrum with legacy RATs which is important to ensure the system feasibility. As a result, the sum rate achieved decreases and as a trade off, the system feasibility rate increases. The performance improvement of D-SRM over D-PCM is greater when the system approaches infeasibility.

Pricing indices are used by the $\mathrm{HG}$ to determine the services' priority for claiming resources. They are updated according to (23) to find a possible feasible allocation when the current allocation is infeasible. However, the number of resource claim iterations is limited by the iteration threshold 
TABLE I

EXEMPLARY ALLOCATION

\begin{tabular}{|c|c|c|c|c|c|c|c|}
\hline \multicolumn{8}{|c|}{ Available Channels in the Allocation } \\
\hline Channel Index & 1 & 2 & 3 & 4 & 5 & 6 & 7 \\
\hline Center Frequency $(\mathrm{GHz})$ & 2.412 & 2.437 & 2.462 & 2.484 & 57.1 & 57.2 & 57.3 \\
\hline Bandwidth(MHz) & 20 & 20 & 20 & 20 & 100 & 100 & 100 \\
\hline \multicolumn{8}{|c|}{ Service Requirements } \\
\hline & Link A & Link B & Link C & Link D & & & \\
\hline Required Data Rate(Mbps) & 1 & 10 & 25 & 60 & & & \\
\hline Power Constraint(mW) & 10 & 60 & 100 & 300 & & & \\
\hline \multicolumn{8}{|c|}{ Channel Allocation Result } \\
\hline & Link A & Link B & Link C & Link D & & & \\
\hline Wi-Fi(30mW) & & 4 & 3,4 & $1,2,3$ & & & \\
\hline $\mathrm{CR}(60 \mathrm{~mW})$ & & & & $5,6,7$ & & & \\
\hline Bluetooth $(10 \mathrm{~mW})$ & $1,2,3,4$ & & & & & & \\
\hline Achieved Data Rate(Mbps) & 1 & 13 & 27 & 76 & & & \\
\hline
\end{tabular}

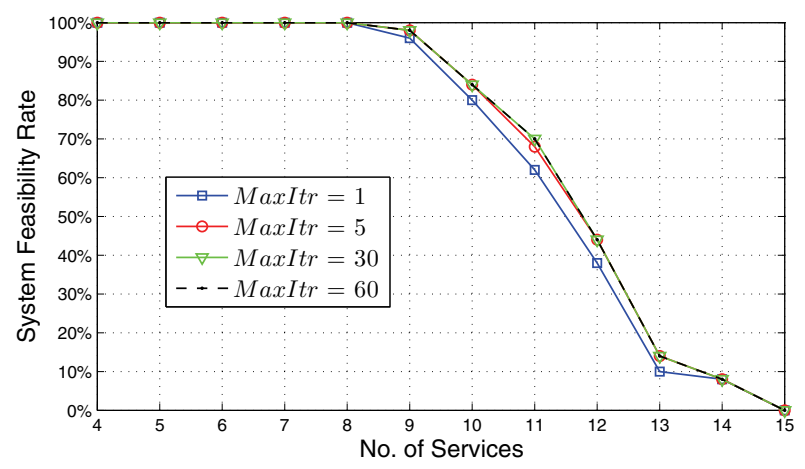

Fig. 8. Convergence of resource claim iterations (D-PCM). The services have same individual rate requirement of $10 \mathrm{Mbps}$. The effect of the iteration threshold MaxItr is shown for the D-PCM algorithm implementation.

MaxItr. In Fig. 8, the impact of MaxItr is shown in terms of system feasibility for D-PCM. 4 channels are considered. The results show that when MaxItr increases from 1 to 5, the performance gain is largest. The reason for this observation is due to our sensible design of heuristic algorithms for exploring the feasibility of system and even within a small number of MaxItr, the proposed algorithm D-PCM could well detect the nature of the system feasibility. Our extensive experiments indicate that MaxItr $=5$ is a reasonable choice for balancing the computation and accuracy.

In the following, the performance of both the D-PCM and D-SRM are evaluated in a more general setting. Rather than identical rate requirement for each service, three classes of services with differing rate requirements exist, i.e. lowrate services, medium-rate services and high-rate services, are considered. Also, the number of services approaches to 20 so that the limiting behaviors of algorithms can be shown. 6 channels are considered while the fraction of each class of service can vary.

In Fig. 9 and Fig. 10, the performance of D-SRM with power control is shown. To illustrate the performance improvement introduced by power control, the individual maximal transmission power is set to $60 \mathrm{~mW}$ for each service while other settings stay the same. The services can use the proposed power control scheme to efficiently assign its transmission power so that channel diversity can be explored. As a result, D-SRM with power control has obvious improvement over

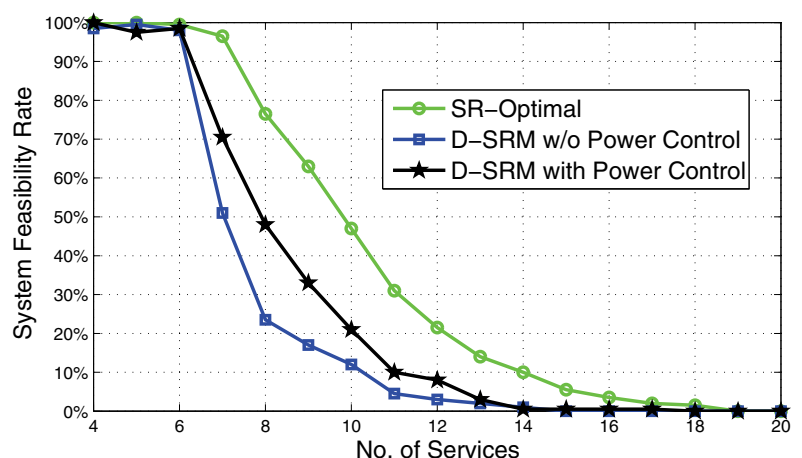

Fig. 9. Power Control (System Feasibility Rate). Results are shown for different service distribution. 6 channels are used. Each service's transmission power is within $60 \mathrm{~mW}$. $P_{\text {wifi }}^{\max }=35 \mathrm{~mW}$ and $P_{C R}^{\max }=65 \mathrm{~mW}$. For each problem instance, the composition of the three classes of services is generated uniformly. Low rate: [5,15]Mbps. Medium rate: $[15,25] \mathrm{Mbps}$. High rate: $[30,40] \mathrm{Mbps}$

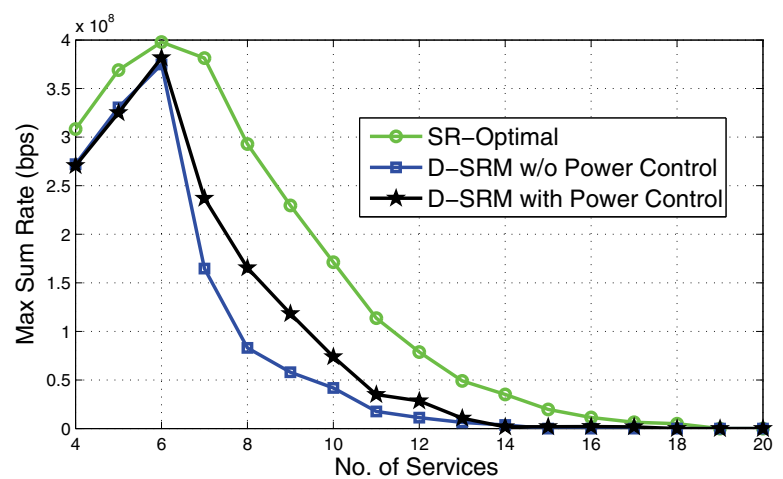

Fig. 10. Power Control (Sum Rate). Results are shown for different service distribution. 6 channels are used. Each service's transmission power is within $60 \mathrm{~mW}$. $P_{\text {wifi }}^{\max }=35 \mathrm{~mW}$ and $P_{C R}^{\max }=65 \mathrm{~mW}$. For each problem instance, the composition of the three classes of services is generated uniformly. Low rate: $[5,15]$ Mbps. Medium rate: $[15,25]$ Mbps. High rate: $[30,40] \mathrm{Mbps}$

that without power control in terms of system feasibility and sum rate both.

When the system is determined infeasible, an admission control scheme is necessary as stated in section III E. In Fig. 11, 6 channels are used for simulation. The number of services varies from 4 to 10 . The same three classes of services described earlier are considered. The SR channel 


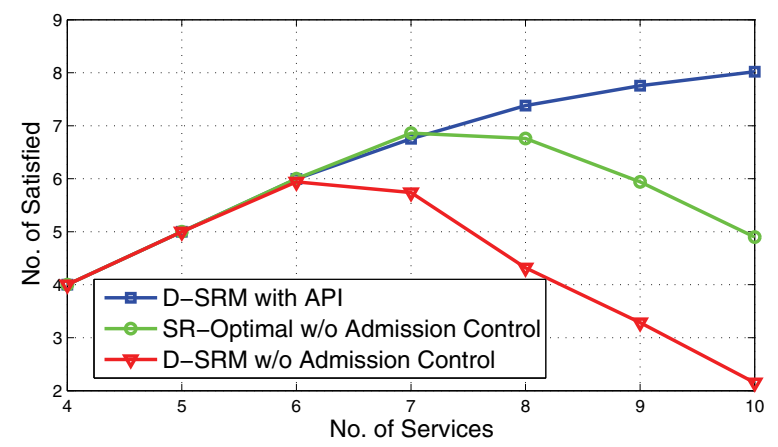

Fig. 11. Admission Control Scheme. Results are shown for different service distribution. 6 channels are used. For each problem instance, the composition of the three classes of services is generated uniformly. Low rate: $[5,15]$ Mbps. Medium rate: $[15,25]$ Mbps. High rate: $[30,40]$ Mbps. Each service's transmission power is within $200 \mathrm{~mW}$.

access model is used. The modified D-SRM algorithm with the API admission control scheme is compared with D-SRM and the optimal solution obtained from the branch and bound method [23]. The simulation result clearly shows that the admission control scheme will help the $\mathrm{CDH}$ support more services than otherwise.

\section{Discussion AND Future WORK}

In this paper, we proposed a framework for distributed resource allocation and admission control in a Cognitive Digital Home (CDH). Two channel access models were considered in the $\mathrm{CDH}$ for addressing spectrum coexistence of legacy devices: (i) Pessimistic Controllability (PC) Model where the HG had no influence over legacy devices, and (ii) Switched RAT (SR) Model where the HG had perfect control of legacy devices. Two resource allocation problems (i) Maximizing Sum Rate, and (ii) Maximizing Service Capacity were solved in a distributed manner to reduce the sensing and computation burden. Distributed algorithms were designed using partial dual decomposition techniques. A distributed power control scheme was developed for efficient use of energy. Based on the pricing information obtained from the distributed algorithms, an admission control scheme was designed to improve the system feasibility. The extensive numerical simulation showed that the proposed resource allocation algorithms could efficiently manage the spectral resources allocation in a $\mathrm{CDH}$. The power control and admission control schemes were also shown to greatly improve the system performance.

While this work presents an initial attempt at managing spectrum coexistence in a $\mathrm{CDH}$, there are many challenging open issues that require further attention such as dynamism and scaling in such networks. Moreover, as more novel data and multimedia services, e.g, home automation, wireless HDMI and smart grid, are introduced into the digital home and new radio access technologies in TV white space and $60 \mathrm{GHz}$ radio, are developed for supporting them, efficient spectrum coexistence of multiple RATs becomes an interesting and urgent task.
APPENDIX

\section{A List of AbBREViations And Notations}

- $\mathrm{CDH}$ Cognitive Digital Home

- HG Home Genie Node

- RAT Radio Access Technology

- MPR Multi-Platform Radio

- CR Cognitive Radio

- MSR Maximizing Sum Rate Problem

- MSC Maximizing Service Capacity Problem

- PC Pessimistic Controllability Model

- SR Switched RAT Model

- API Average Pricing Index admission control scheme

- D-PCM Distributed Algorithm for PC-MSR(PC-MSC)

- D-SRM Distributed Algorithm for SR-MSR(SR-MSC)

- GSM Global Spectrum Map

- $\mathcal{K}$ the set of services

- $\mathcal{M}$ the set of channels

- $\mathcal{T}$ the set of RATs

- $x(k, i, t)$ the indicator whether service $k$ occupies channel $i$ with RAT $t$

- $l(k, i, t)$ the portion of channel for service $k$ on channel $i$ with RAT $t$
- $P(k, i, t)$ the service $k$ 's transmit power on channel $i$ with RAT $t$

- $R^{P}(k, i, t)$ the theoretical physical rate of service $k$ on channel $i$ with RAT $t$

- $R(k, i, t)$ the effective rate service $k$ can achieve on channel $i$ with RAT $t$

- $\mathbf{X}$ the $k$-th row is $\mathbf{x}_{\mathbf{k}}$ as in (2)

- $\mathbf{L}$ the $k$-th row is $\mathbf{l}_{\mathbf{k}}$ as in (3)

- $w_{i}$ bandwidth of channel $i$

- $h_{k}^{i}$ service $k$ 's channel gain on channel $i$

- $N_{0}$ noise level

- $R_{k}^{\text {min }}$ service $k$ 's minimum data rate requirement

- $P_{t}$ nominal transmit power for RAT $t$

- $P_{k}^{\max }$ service $k$ 's maximal transmit power

- $P I_{k}(i t r)$ service $k$ 's pricing index in $i t r$-th claim iteration

- $P_{t}^{\max } \mathrm{RAT} t$ 's maximal transmit power

- MaxItr the threshold for the number of resource claim iterations

\section{REFERENCES}

[1] IEEE, "IEEE Standard for Information TechnologyTelecommunications and information exchange between systems Wireless Regional Area Networks (WRAN)-Specific requirements Part 22: Cognitive Wireless RAN Medium Access Control (MAC) and Physical Layer (PHY) Specifications: Policies and Procedures for Operation in the TV Bands," July 2011.

[2] C. R. Stevenson, G. Chouinard, Z. Lei, W. Hu, S. J. Shellhammer, and W. Caldwell, "IEEE802.22: the first cognitive radio wireless regional area networks standard," IEEE Commun. Mag., pp. 130-138, Jan. 2009.

[3] IEEE, "IEEE Standard for Information TechnologyTelecommunications and information exchange between systems Local and metropolitan area networks-Specific requirements Part 11: Wireless LAN Medium Access Control (MAC) and Physical Layer (PHY) Specifications," Mar. 2012.

[4] P. Smulders, "Exploiting the $60 \mathrm{GHz}$ band for local wireless multimedia access: prospects and future directions," IEEE Commun. Mag., pp. 140148, Jan. 2002.

[5] M. Peter, W. Keusgen, and J. Luo, "A survey on $60 \mathrm{GHz}$ broadband communication: capability, applications and system design," in Proc. 2008 European Microwave Integrated Circuits Conf., pp. 1-4.

[6] O. Sallent, L. Giupponi, J. Nasreddine, R. Agusti, and J. Perez-Romero, "Spectrum and radio resource management," IEEE Veh. Technol. Mag., vol. 3, no. 4, pp. 56-64, Dec. 2008.

[7] D. E. Charilas and A. D.Panagopoulos, "Multiaccess radio network environment," IEEE Veh. Technol. Mag., vol. 5, no. 4, pp. 40-49, Dec. 2010.

[8] A. Bazzi, G. Pasolini, and O. Andrisano, "Multiradio resource management: paralell transmission for higher throughput," EURASIP J. Advances Signal Process., vol. (2008), 2008.

[9] S. Merlin, N. Vaidya, and M. Zorzi, "Resource allocation in multiradio multi-channel multi-hop wireless networks," in Proc. 2008 IEEE INFOCOM, pp. 610-618.

[10] Y. Choi, H. Kim, S. Han, and Y. Han, "Joint resource allocation for paralell multi-radio access in heterogeneous wireless networks," IEEE Trans. Wireless Commun., vol. 9, no. 11, pp. 3324-3329, Nov. 2010.

[11] S. Kawade and M. Nekovee, "Can cognitive radio access to TV white spaces support future home networks?" in Proc. 2010 IEEE Symp. New Frontiers Dynamic Spectrum, pp. 1-8.

[12] T. Li, N. B. Mandayam, and A. Reznik, "A framework for resource allocation in a cognitive digital home," in Proc. 2010 IEEE Global Commun. Conf., pp. 1-5. 
[13] _ - "Distributed algorithms for joint channel and RAT allocation in a cognitive digital home," in Proc. 2011 Int. Symp. Modeling Optimization Mobile, Ad Hoc, Wireless Netw., pp. 213-219.

[14] C. Won, J. Youn, H. Ali, H. Sharif, and J. Deogun, "Adaptive radio channel allocation for supporting coexistence of 802.15.4 and 802.11b," in Proc. 2005 IEEE Veh. Technol. Conf. - Fall, pp. 2522-2526.

[15] C. Raman, R. D. Yates, and N. B. Mandayam, "Scheduling variable rate links via a spectrum server," in Proc. 2005 IEEE DySPAN, pp. 110-118.

[16] Z. Miljanic, I. Seskar, K. Le, and D. Raychaudhuri, "The WINLAB network centric cognitive radio hardware platform - WiNC2R," MONET Special Issue Cognitive Radio Oriented Wireless Netw. Commun., vol. 13, no. 5, pp. 533-541, Oct. 2008.

[17] R. Rajbanshi, A. M. Wyglinski, and G. Minden, "An efficient implementation of NC-OFDM transceivers for cognitive radios," in Proc. 2006 Int. Conf. Cognitive Radio Oriented Wireless Netw. Commun., pp. 1-5.

[18] C. F. Chiasserini and R. R. Rao, "Performance of IEEE 802.11 WLANs in a bluetooth environment," in Proc. 2000 IEEE Wireless Commun. Netw. Conf., pp. 94-99.

[19] — "Coexistence mechanisms for interference mitigation in the 2.4GHz ISM band," IEEE Trans. Wireless Commun., Sept. 2003.

[20] A. Sikora and V. F. Groza, "Coexistence of IEEE802.15.4 with other systems in the $2.4 \mathrm{GHz}-\mathrm{ISM}$-band," in Proc. 2005 IEEE IMTC.

[21] S. C. Liew, C. H. Kai, H. C. Leung, and P. Wong, "Back-of-the-envelope computation of throughput distributions in CSMA wireless networks," IEEE Trans. Mobile Comput., vol. 5, no. 5, pp. 1319-1331, Sept. 2010.

[22] C. Gomez and J. Paradells, "Wireless home automation networks: a survey of architectures and technologies," IEEE Commun. Mag., no. 6, pp. 92-101, June 2010.

[23] L. A. Wolsey and G. L. Nemhauser, Integer and Combinatorial Optimization. John Wiley and Sons, Inc., 1999.

[24] C. Chekuri and S. Khanna, "A PTAS for the multiple knapsack problem," in Proc. 2010 Ann. ACM-SIAM Symp. Discrete Algorithms, pp. 213-222.

[25] D. Palomar and M. Chiang, "A tutorial on decomposition methods for network utility maximization," IEEE J. Sel. Areas Commun., vol. 24, no. 8, pp. 1439-1451, Aug. 2006.

[26] A. Ruszczynski, Nonlinear Optimization. Princeton University Press, 2006.

[27] J. W. Chinneck, Feasibility and Infeasibility in Optimization. Springer, LLC., 2008.

[28] E. Amaldi, M. E. Pfetsch, and L. Trotter Jr., "Some structural and algorithmic properties of the maximum feasible subsystem problem," in Proc. 2000 Integer Programming Combinatorial Optimization Conf., 2000.

[29] J. W. Chinneck, "An effective polynomial-time heuristic for the minimum-cardinality IIS set-covering problem," Ann. Math. Artificial Intell., pp. 127-144, 1996.

[30] T. S. Rappaport, Wireless Communications: Principles and Practice. Prentice Hall, 1996.

[31] N. Moraitis and P. Constantinou, "Indoor channel measurements and characterization at $60 \mathrm{GHz}$ for wireless local area network applications," IEEE Trans. Antennas Propag., no. 12, pp. 3180-3189, Dec. 2004.

[32] A. Goldsmith, Wireless Communications. Cambridge University Press, 2005.

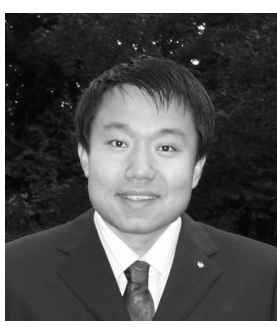

Tianming Li received his B.S. degree from Shanghai Jiao Tong University, Shanghai, China in 2006 and his M.S. degree from the University of New Mexico, Albuquerque, USA in 2008, both in electrical engineering. $\mathrm{He}$ is currently a $\mathrm{Ph} . \mathrm{D}$. candidate at the WINLAB, Department of Electrical and Computer Engineering at Rutgers University. His research interests include efficient resource allocation and management in wireless communication networks. His previous research work included dynamic spectrum leasing in cognitive radio networks.

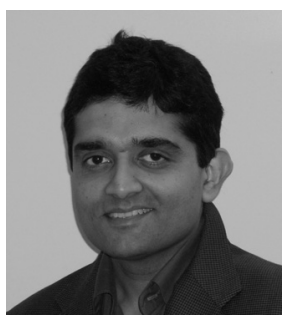

Narayan B. Mandayam is currently the Peter D. Cherasia Faculty Scholar at Rutgers University. He received the B.Tech (Hons.) degree in 1989 from the Indian Institute of Technology, Kharagpur, and the M.S. and Ph.D. degrees in 1991 and 1994 from Rice University, all in electrical engineering. From 1994 to 1996, he was a Research Associate at the Wireless Information Network Laboratory (WINLAB), Rutgers University before joining the faculty of the Electrical and Computer Engineering department at Rutgers where he became Associate Professor in 2001 and Professor in 2003. Currently, he also serves as Associate Director at WINLAB. He was a visiting faculty fellow in the Department of Electrical Engineering, Princeton University in 2002 and a visiting faculty at the Indian Institute of Science in 2003. His research interests are in various aspects of wireless data transmission with emphasis on techniques for cognitive radio networks including their implications for spectrum policy. Using constructs from game theory, communications and networking, his work focuses on system modeling and performance, signal processing as well as radio resource management for enabling wireless technologies to support various applications.

Dr. Mandayam is a recipient of the Fred W. Ellersick Prize from the IEEE Communications Society in 2009 along with O. Ileri for their work on dynamic spectrum access models and spectrum policy. He is also a recipient of the Institute Silver Medal from the Indian Institute of Technology in 1989 and the National Science Foundation CAREER Award in 1998. He is a coauthor of the books, Principles of Cognitive Radio (Cambridge, 2012) and Wireless Networks: Multiuser Detection in Cross-Layer Design (Springer, 2005). He has served as an Editor for the journals IEEE COMMUNICATION LETTERS and IEEE TRANSACTIONS ON WIRELESS COMMUNICATIONS. He has also served as a guest editor of the IEEE JSAC Special Issues on Adaptive, Spectrum Agile and Cognitive Radio Networks (2007) and Game Theory in Communication Systems (2008). He is a Fellow of the IEEE and currently serves as a Distinguished Lecturer of the IEEE Communications Society.

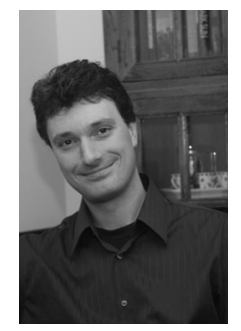

Alex Reznik is a Senior Principal Engineer at InterDigital, currently leading the company's research and system design activities in the area of IP mobility and heterogeneous networks. Since joining InterDigital in 1999, he has been involved in a wide range of projects, including leadership of $3 \mathrm{G}$ modem ASIC architecture, design of advanced wireless security systems, and coordination of standards strategy in the cognitive networks space. He earned his B.S.E.E. Summa Cum Laude from The Cooper Union, S.M. in Electrical Engineering and Computer Science from the Massachusetts Institute of Technology, and Ph.D. in Electrical Engineering from Princeton University. He holds a visiting faculty appointment at WINLAB, Rutgers University, where he collaborates on research in cognitive radio, wireless security, and future mobile Internet. $\mathrm{He}$ is an inventor of over 60 granted U.S. patents, and has been awarded numerous awards for Innovation at InterDigital. 